\title{
Branched fatty acids inhibit the biosynthesis of menaquinone in Helicobacter pylori
}

\author{
Ruri Tanaka $^{1}$, Takao Kunisada ${ }^{2}$, Nobuaki Kushida ${ }^{3}$, Keiko Yamada ${ }^{3}$, Shunsuke Ikeda ${ }^{4}$, Motoyoshi Noike ${ }^{1,4}$, \\ Yuusuke Ono ${ }^{1,4}$, Nobuya Itoh $^{1}$, Hideto Takami ${ }^{5}$, Haruo Seto ${ }^{6}$ and Tohru Dairi ${ }^{1,4}$
}

The Journal of Antibiotics (2011) 64, 151-153; doi:10.1038/ja.2010.133; published online 17 November 2010

Keywords: actinomycetes; fatty acid; futalosine pathway; inhibitor

Menaquinone (MK) is an essential compound because it is an obligatory component of the electron transfer pathway in microorganisms. In Escherichia coli, MK was shown to be derived from chorismate by eight enzymes, designated MenA-H $\mathrm{H}^{1,2}$ However, we have revealed that an alternative pathway (we named it the futalosine pathway; Figure 1$)^{3-5}$ was operating in some microorganisms including Helicobacter pylori, which causes gastric carcinoma. As humans and some useful intestinal bacteria, such as lactobacilli, possess the classical pathway, and MK biosynthesis is essential for survival of microorganisms ${ }^{4}$ the futalosine pathway is an attractive target for the development of specific anti-H. pylori drugs. In this study, we tried to obtain such compounds from metabolites produced by actinomycetes and fungi.

To identify compounds that specifically inhibit the futalosin pathway, we developed a screening method. We previously showed that the MqnA-D genes in the futalosine pathway were essential for survival, as these gene-disrupted Streptomyces coelicolor strains required exogenously added MK for their growth. Therefore, a compound that inhibits the growth of $S$. coelicolor but does not inhibit its growth in the presence of MK would become a candidate. However, this assay method is laborious and the growth of S. coelicolor is too slow to screen a mass of samples. Therefore, we employed a paper disk-agar diffusion assay, which is based on the phenomenon that antibiotics will diffuse from a paper disk into an agar medium containing test organisms and form a growth-inhibitory zone. We used two kinds of Bacillus strains as test organisms. One was Bacillus subtilis and the other is $B$. halodurans C-125. By genome sequencing, the latter strain was shown to be quite similar to the former strain in terms of genome size, $\mathrm{G}+\mathrm{C}$ content of genomic DNA and the physiological properties used for taxonomical identification. ${ }^{6}$ Moreover, the phylogenetic placement of B. halodurans C-125 based on 16S rDNA sequence analysis indicated that this organism is more closely related to B. subtilis than to other members of the genus Bacillus. ${ }^{6}$ For example, both strains showed similar MIC values against representative antibiotics except for clarithromycin (Table 1). The resistance to clarithromycin was probably caused by the presence of an ermD gene that encodes the ribosome-methylation enzyme in B. halodurans. ${ }^{7}$ However, judging from the genome database of these strains, B. subtilis and B. halodurans $\mathrm{C}-125$ use the classical pathway and the futalosine pathway, respectively, for the biosynthesis of MK. ${ }^{6}$ These facts suggested that a compound inhibiting the biosynthesis of MK in the futalosine pathway specifically represses the growth of only B. halodurans $\mathrm{C}-12$. Therefore, we first screened candidate compounds for their ability to specifically inhibit $B$. halodurans C-125 using a paper disk assay. We tested approximately 1800 culture broths (1000 actinomycetes broths and 800 fungi broths). Of these, approximately 300 culture broths (17\%) formed growth-inhibitory zone against both B. subtilis and B. halodurans $\mathrm{C}-125$. However, we found that two actinomycetes culture broths specifically inhibited the growth of $B$. halodurans $\mathrm{C}-125$ (hit ratio, $0.1 \%$ ) (Figure 2). Then we examined whether B. halodurans C-125 could recover from this inhibition when MK $\left(0.1 \mathrm{mg} \mathrm{ml}^{-1}\right)$ was added into the culture broth during liquid cultivation. The growth of B. halodurans C-125 was clearly inhibited in the presence of sample no. AF50404, but this inhibition was reversed by adding MK, even in the presence of sample no. AF50404. This result strongly suggested that sample no. AF50404 contained a compound that specifically inhibited the futalosine pathway. The other candidate (AF50573) also showed the same inhibitory phenotype as that of no. AF50404, but it gradually lost its activity, probably because of its instability. Therefore, we used sample no. AF50404 in further analyses.

${ }^{1}$ Biotechnology Research Center, Toyama Prefectural University, Toyama, Japan; ${ }^{2}$ Bioscience Laboratories, Meiji Seika Kaisha, Kanagawa, Japan; ${ }^{3}$ Pharmaceutical Research Center, Meiji Seika Kaisha, Yokohama, Japan; ${ }^{4}$ Laboratory of Applied Biochemistry, Graduate School of Engineering, Hokkaido University, Hokkaido, Japan; ${ }^{5}$ Microbial Genome Research Group, Japan Agency for Marine-Earth Science and Technology, Yokosuka, Japan and ${ }^{6}$ Faculty of Applied Bio-science, Tokyo University of Agriculture, Setagaya-ku, Japan

Correspondence: Professor T Dairi, Laboratory of Applied Biochemistry, Graduate School of Engineering, Hokkaido University, Hokkaido 060-8628, Japan.

E-mail: dairi@eng.hokudai.ac.jp

Dedicated to the late $\operatorname{Dr} \mathrm{C}$ Richard Hutchinson for his exceptional contributions to natural product biosynthesis, engineering and drug discovery.

Received 10 August 2010; revised 12 October 2010; accepted 13 October 2010; published online 17 November 2010 


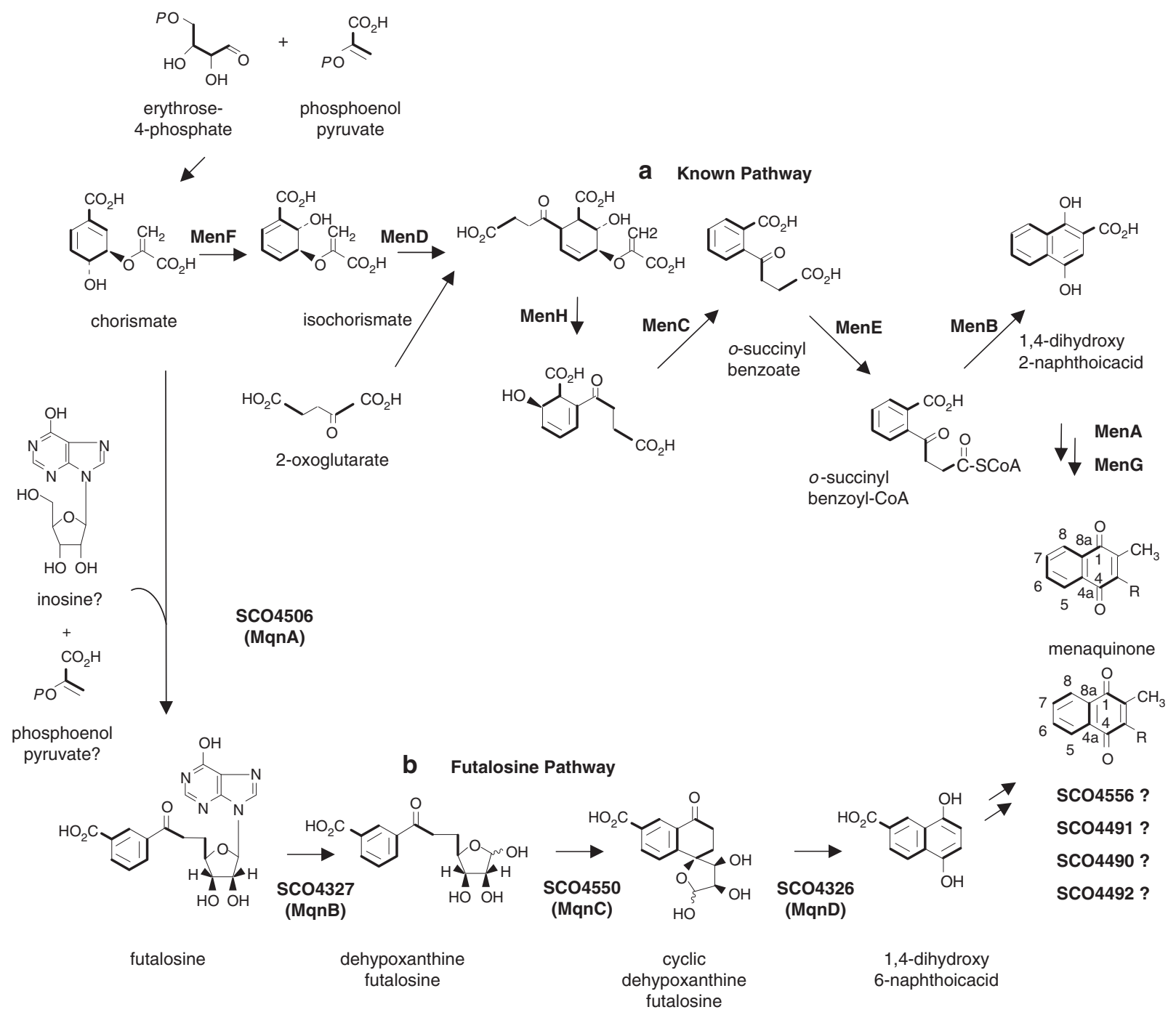

Figure 1 Menaquinone biosynthetic pathway. (a) Known pathway, (b) futalosine pathway.

Table 1 MIC of some antibiotics against $B$. subtilis and $B$. halodurans

\begin{tabular}{lccccc}
\hline & \multicolumn{5}{c}{ Compounds $\left(M / C\left(\mu g\right.\right.$ I $\left.\left.^{-1}\right)\right)$} \\
\cline { 2 - 6 } Strain & Clarithromycin & Kanamycin & Ampicillin & Tetracycline & Ciprofloxacin \\
\hline B. subtilis & 0.06 & 1 & 0.015 & 4 & 0.12 \\
B. halodurans & $>128$ & 4 & 0.25 & 0.12 & 0.06 \\
\hline
\end{tabular}

We purified active compounds from sample no. AF50404 that inhibited the futalosine pathway. The actinomycete that was used for the preparation of sample no. AF50404 was cultivated in a 100-ml Erlenmeyer flask containing $15 \mathrm{ml}$ seed medium (starch $2.5 \%$, glucose $2.0 \%$, polypeptone $0.7 \%$, wheat germ $0.6 \%$, yeast extract $0.45 \%$, soybean meal $0.3 \%$, Lab-Lemco-Powder (Merck) $0.3 \%, \mathrm{CaCO}_{3}$ $0.2 \%, \mathrm{pH} 7.1)$ on a rotary shaker ( 200 r.p.m.) at $28^{\circ} \mathrm{C}$ for 4 days. A portion of the medium was transferred into a 500-ml Erlenmeyer flask containing $80 \mathrm{ml}$ of production medium (malt syrup 4.0\%, soybean meal $2.0 \%$, cotton seed lees $1.0 \%, \mathrm{FeSO}_{4} 0.001 \%, \mathrm{CoCl}_{2} 0.0001 \%$, $\mathrm{NiCl}_{2} 0.0001 \%, \mathrm{pH} 6.0$ ) and cultivated for 8 days. After cultivation, $80 \mathrm{ml}$ acetone was added into the flask and the flask was placed on shaker for $1 \mathrm{~h}$. After filtration, the acetone was removed by evaporation and the resulting aqueous layer (in total, 51 broth) was used for further purification. The aqueous layer was extracted with the same volume of ethyl acetate at neutral $\mathrm{pH}$ and concentrated in vacuo. The dried material was dissolved in a small volume of methanol and subjected to Diaion HP20 resin (Mitsubishi Chemical Corporation, Tokyo, Japan) column chromatography (bed volume, $200 \mathrm{ml}$ ); then the column was washed with $70 \%$ methanol (21). The materials were eluted with $400 \mathrm{ml}$ methanol and evaporated to dryness under reduced pressure. The dried material was dissolved in a small volume of methanol and then fractionated by preparative HPLC (column; Merck Mightysil RP-18 column $(250 \times 20 \mathrm{~mm})$; mobile phase, $50-100 \%$ acetonitrile linear gradient; flow rate, $5 \mathrm{ml} \mathrm{min}^{-1}$; detection, $210 \mathrm{~nm}$ ). The combined active fractions underwent structural analysis. The ${ }^{1} \mathrm{H}$-NMR spectrum indicated that the material was a mixture of fatty acids branched at the alkyl terminus. The major component showed one doublet at $\delta 0.84$ and one triplet at $\delta 0.85$, and the minor component showed one doublet at $\delta 0.86$. These data, combined with the triplet at $\delta 2.35$ and multiplets at $\delta 1.0-1.7$, supported the argument that these compounds were branched fatty acids. GC-MS analysis indicated that the compounds were two C15 saturated fatty 


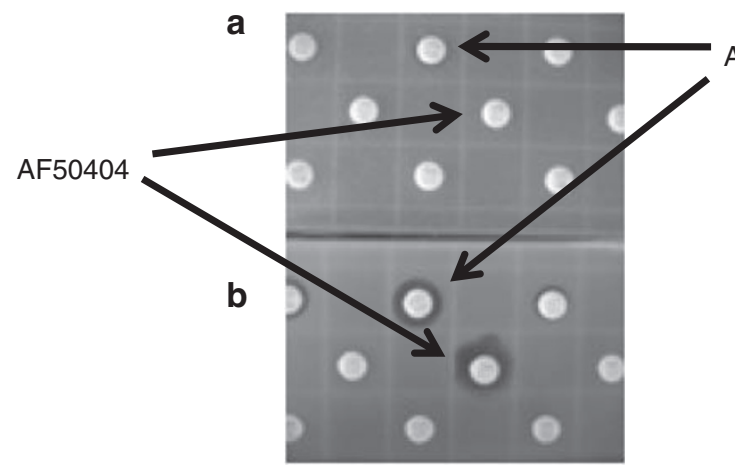

AF50573

Figure 2 Bioassay with paper disks. B. subtilis (a) and B. halodurans (b) were used as test microorganisms.

a<smiles>CCC(C)CCCCCCCCCCC(=O)O</smiles>

b<smiles>CC(C)CCCCCCCCCCCC(=O)O</smiles><smiles>CC(C)=CCC/C(C)=C/CC/C(C)=C/CC/C(C)=C/CC1=C(C)C(=O)c2ccccc2C1=O</smiles>

Figure 3 Structures of 12-methyltetradecanoic acid (a), 13-methyltetradecanoic acid (b) and menaquinone 4 (c).

acids (8:2, MW 242). Therefore, we purchased the estimated C15 saturated fatty acids and compared their physicochemical properties (retention time and mass spectral fragmentation patterns) by GC-MS and antibacterial activities with those of the purified sample. Consequently, we confirmed that the active compounds were 12-methyltetradecanoic acid (main component) and 13-methyltetradecanoic acid (minor component) (Figure 3). The commercially obtained 12methyltetradecanoic acid alone showed almost the same growthinhibitory effect as the purified natural mixed products against B. halodurans C-125. The same result was also obtained with the purchased 13-methyltetradecanoic acid, suggesting that the growthinhibiting effect of the natural mixed products did not reflect a synergistic effect of both compounds.

We next examined whether these two compounds indeed inhibited the growth of $H$. pylori. H. pylori ATCC43504 was cultivated on Mueller-Hinton agar (Becton Dickinson and Company, Franklin Lakes, NJ, USA) containing 5\% sheep defibrinated blood (MHA agar) at $35^{\circ} \mathrm{C}$ for $72 \mathrm{~h}$. The cells were collected and adjusted to McFarland standard turbidity no. 2 with saline. The prepared cells were spread on MHA agar containing various concentrations of 12-methyltetradecanoic acid or 13-methyltetradecanoic acid, which were dissolved in dimethyl sulfoxide to a concentration of $12.8 \mathrm{mg} \mathrm{ml}^{-1}$ and serially diluted. As shown in Table 2, both compounds had an MIC of $32 \mu \mathrm{g} \mathrm{ml}^{-1}$. These values were high when compared with clarithromycin, which is usually used for treating $H$. pylori-positive patients, but significantly lower than those of other fatty acids, such as palmitic acid and stearic acid used as negative controls $\left(>128 \mu \mathrm{g} \mathrm{ml}^{-1}\right)$.
Table 2 MIC of various fatty acids against $H$. pylori ATCC43504

\begin{tabular}{lc} 
Compounds & MIC $\left(\mu \mathrm{g} \mathrm{ml^{-1 }}\right)$ \\
\hline Clarithromycin & 0.015 \\
12-Methyltetradecanoic acid & 32 \\
13-Methyltetradecanoic acid & 32 \\
Pentadecanoic acid & $>128$ \\
Palmitic acid & $>128$ \\
Stearic acid & $>128$
\end{tabular}

Clarithromycin and pentadecanoic acid/palmitic acid/stearic acid were used as positive and negative control, respectively.

We next tried to examine which biosynthetic step in the futalosine pathway was inhibited by the fatty acids. However, we did not have sufficiently large amounts of the intermediate compounds in the futalosine pathway. The only compound available for the experiment was futalosine, the first intermediate compound in the futalosine pathway. Therefore, we examined whether the growth of $B$. halodurans C-125 recovered when futalosine $\left(0.1 \mathrm{mg} \mathrm{ml}^{-1}\right)$ was added in the medium containing 12-methyltetradecanoic acid or 13-methyltetradecanoic acid. B. halodurans $\mathrm{C}-125$ was not able to grow in the presence of both the saturated fatty acid and futalosine. This result suggested that the fatty acids probably inhibited a step after the formation of futalosine. Considering the structural similarity between the prenyl side chain of MK and 12/13-methyltetradecanoic acid (Figure 3), a possible target might be an enzyme that catalyzes the transfer of prenyl side chain into naphthoquinone moiety.

To date, several studies showing that some fatty acids inhibit the growth of $H$. pylori have been reported. ${ }^{89}$ However, their mode of action remains unclear. In this study, it was shown that 12-methyltetradecanoic acid and 13-methyltetradecanoic acid perhaps inhibit a step after the formation of futalosine.

\section{ACKNOWLEDGEMENTS}

We thank Dr T Ida of Meiji Seika Kaisha Ltd for the measurement of sensibilities to standard antibiotics of $B$. subtilis and B. halodurans. This work was supported in part by a Grant-in-Aid for Scientific Research (B) from the Ministry of Education, Culture, Sports and Technology of Japan and Institute for Fermentation, Osaka (IFO) to TD.

1 Bentley, R. \& Meganathan, R. Biosynthesis of vitamin K (menaquinone) in bacteria. Microbiol. Rev. 46, 241-280 (1982).

2 Meganathan, R. Biosynthesis of menaquinone (vitamin K2) and ubiquinone (coenzyme Q): a perspective on enzymatic mechanisms. Vitam. Horm. 61, 173-218 (2001).

3 Seto, H. et al. Studies on a new biosynthetic pathway for menaquinone. J. Am. Chem. Soc. 130, 5614-5615 (2008).

4 Hiratsuka, T. et al. An alternative menaquinone biosynthetic pathway operating in microorganisms. Science 321, 1670-1673 (2008).

5 Hiratsuka, T., Itoh, N., Seto, H. \& Dairi, T. Enzymatic properties of futalosine hydrolase, an enzyme essential for a newly identified menaquinone biosynthetic pathway. Biosci. Biotech. Biochem. 73, 1137-1141 (2009).

6 Takami, H. et al. Complete genome sequence of alkaliphilic bacterium, Bacillus halodurans and genomic sequence comparison with Bacillus subtilis. Nucleic Acids Res. 28, 4317-4331 (2000).

7 Bozdogam, B., Galopin, S. \& Leclercq, R. Characterization of a new erm-related macrolide resistance gene present in probiotic strains of Bacillus clausii. Appl. Environ. Microbiol. 70, 280-284 (2004).

8 Thompson, L., Cockayne, A. \& Spiller, R. C. Inhibitory effect of polyunsaturated fatty acids on the growth of Helicobacter pylori: a possible explanation of the effect of diet on peptic ulceration. Gut 35, 1557-1561 (1994).

9 Roberton, A. M., O'Connor, C. J., Martin, I. G. \& Alany, R. G. Gastric therapies and compositions thereof. JP 2008-510804A (WO2006/022560). 